\title{
Qualidade de vida dos graduandos de enfermagem e a relação entre os dados sociodemográficos
}

\section{Quality of life of nursing students and the relationship between socio- demographic data}

\author{
Camila Scarel Rosa \\ Universidade Federal de São Paulo - UNIFESP - São Paulo - Brasil \\ $\underline{\text { mila_sr@msn.com }}$ \\ Grazielle Viola Arronqui \\ Universidade Federal de São Paulo - UNIFESP - São Paulo - Brasil \\ grazi.viola@gmail.com \\ Rosely Erlach Goldman \\ Universidade Federal de São Paulo - UNIFESP - São Paulo - Brasil \\ rosely.goldman@unifesp.br
}

\section{RESUMO}

OBJETIVO: O presente estudo teve por objetivo conhecer a percepção de graduandos de enfermagem sobre sua qualidade de vida e correlacionar aos dados sociodemográficos.

MÉTODOS: Estudo transversal, exploratório, realizado na Escola Paulista de Enfermagem (EPEUNIFESP). A amostra foi constituída por 185 graduandos. Os dados foram coletados com questionário contendo perguntas fechadas referentes às variáveis sociodemográficas e o instrumento genérico WHOQOL-bref para a qualidade de vida.

RESULTADOS: Os resultados demostram que os graduandos consideraram sua qualidade de vida boa. No domínio relações sociais há o maior escore médio, o menor foi obtido para o meio ambiente. Na correlação com variáveis sociodemográficas, a renda per capita familiar mensal, os contribuintes e dependentes da renda, o nível de apoio recebido para cursar enfermagem e a presença de crença se mostraram significantes na promoção da qualidade de vida.

CONCLUSÕES: Concluiu-se que variáveis sociodemográficas relacionadas ao aspecto financeiro, o nível de apoio recebido dos pais e amigos para cursar enfermagem e a religiosidade influenciaram o julgamento dos graduandos em relação à satisfação com as facetas avaliadas pelo WHOQOL-bref, contribuindo para obtenção de resultados elevados nos diferentes domínios.

PALAVRAS-CHAVES: Qualidade de vida. Enfermagem. Estudante de enfermagem.

\section{ABSTRACT}

OBJECTIVE: This study aimed to know the perception of nursing students on their quality of life and correlate to sociodemographic data. 
METHODS: Cross-sectional, exploratory, and held in São Paulo School of Nursing (EPEUNIFESP). The sample consisted of 185 undergraduate students Data were collected with a questionnaire containing closed questions regarding sociodemographic variables and the generic instrument WHOQOL-bref for quality of life.

RESULTS: The results shows that undergraduates considered their quality of life good. Social relations are the highest and the lowest mean score was obtained for the environment. In correlation with sociodemographic variables, per capita family income, taxpayers and dependents of income, the level of support received to study nursing and the presence of belief proved to be significant in promoting quality of life.

CONCLUSIONS: It was concluded that sociodemographic variables related to the financial aspect, the level of support received from parents and friends to attend nursing and religiosity influenced the judgment of students in relation to their satisfaction with the facets assessed by the WHOQOL$\mathrm{BREF}$, contributing to achieving results elevated in different domains.

KEYWORDS: Quality of life. Nursing. Nursing Student.

\section{Introdução}

A qualidade de vida (QV) é conceito que permanece em ênfase nesta última década. Desde sua primeira citação por Lyndon Johnson, em 1964, até os dias de hoje, sofreu grandes mudanças em sua definição. O termo, inicialmente, foi utilizado apenas sob aspectos objetivos - como descritor de aquisição de bens materiais. Com o passar dos anos foi ampliado, tornando-se multifacetário. Atualmente, abrange também os aspectos subjetivos, sendo constituído de inúmeras competências e grandezas, como: ambientais, físicas, psicológicas, espirituais, entre outras (FLECK et al., 1999).

O grupo de QV da Organização Mundial da Saúde, sob a coordenação de John Orley, definiu QV como "[...] a percepção do indivíduo de sua posição na vida no contexto da cultura e sistema de valores nos quais ele vive e em relação aos seus objetivos, expectativas, padrões e preocupações" (WHOQOL GROUP, p. 1405, 1995).

A preocupação com a QV pode estar relacionada à aprendizagem, ao interesse e à motivação, como também com problemas financeiros, familiares e de saúde (SAUPE, 2002; ALVES, 2010). Ao passo que, o ingresso do estudante no curso superior requer adaptações à nova realidade. As exigências da universidade no âmbito pessoal, social, acadêmico e comportamental podem influenciar direta ou indiretamente na QV do graduando (ALVES, 2010; SAUPE et al., 2004; OLIVEIRA, CIAMPONE, 2006).

Do mesmo modo, a iniciação das atividades práticas pelo graduando em enfermagem é descrito, em alguns estudos, como o período em que ele apresenta menor índice de QV (KAWAKAME; MIYADAHIRA, 2005; OLIVEIRA; CIAMPONE, 2008). Fato que, segundo estes autores, pode estar relacionado ao impacto da inserção em uma realidade a qual não pertence, pela introdução de atividades práticas e pelo contato com pessoas pertencentes a outros estratos sociais.

Assim, no processo de formação do profissional enfermeiro, cuja atuação possui como base o cuidado ao próximo, o conceito de QV é enfatizado como promotor da saúde e do autocuidado. Para ser capaz de cuidar do outro é necessário primeiramente cuidar de si e entender a saúde como um conceito amplo: "[...] um estado dinâmico completo de bem-estar físico, mental, espiritual e social e não meramente a ausência de doença" (WHO/MAS/MHP/98.2, 1998).

Diante do contexto apresentado, o estudo teve como objetivo conhecer a percepção de graduandos de enfermagem sobre sua QV, correlacionando-a com os dados sociodemográficos. 


\section{Método}

Trata-se de estudo descritivo, exploratório, transversal e de natureza quantitativa. A amostra foi constituída de 185 alunos regularmente matriculados, no ano letivo de 2009, da primeira à quarta série do curso de graduação em enfermagem da Escola Paulista de Enfermagem da Universidade Federal de São Paulo (EPE-UNIFESP).

A coleta de dados foi realizada em sala de aula, no campus São Paulo, nos meses de novembro e dezembro de 2009, após aprovação do projeto de pesquisa pelo Comitê de Ética em Pesquisa da Universidade Federal de São Paulo - Protocolo n ${ }^{\circ}$ 0186/09 e autorização da Chefia da Escola de Enfermagem. A participação dos estudantes ocorreu de forma voluntária, formalizada por meio da assinatura individual do Termo de Consentimento Livre e Esclarecido.

Para coleta de dados utilizou-se um questionário com perguntas fechadas referentes às variáveis sociodemográficas e para a QV foi aplicado o instrumento genérico WHOQOL-bref.

O WHOQOL-bref é composto por quatro domínios: físico, psicológico, relações sociais e meio ambiente. O domínio físico explora as facetas de dor e desconforto, energia e fadiga, sono e repouso, mobilidade, atividades da vida cotidiana, dependência de medicações ou tratamentos e capacidade de trabalho do indivíduo. O domínio psicológico avalia sentimentos positivos: pensar, aprender, memória e concentração, autoestima, imagem corporal e aparência; sentimentos negativos e espiritualidade do indivíduo. O domínio das relações sociais abrange relações pessoais, suporte ou apoio social e atividade sexual do indivíduo. O domínio meio ambiente contempla as facetas: segurança física e proteção, ambiente do lar, recursos financeiros, cuidados de saúde e sociais, oportunidades de adquirir novas informações e habilidades, participação e oportunidades de recreação/lazer, ambiente físico e transporte do indivíduo.

Os dados coletados foram transportados para uma planilha do Programa Excel for Windows XP e, posteriormente, ao Programa Statistical Package for the Social Sciences for Windows em sua versão 19.0. Os testes aplicados foram o de normalidade de Shapiro-Wilk e o t de Students para amostras não pareadas com nível de confiança em $95 \%$ e os testes não paramétricos Wilcoxon Matched Pairs Test com nível de significância $\mathrm{p}<0,05$.

\section{Resultados}

Do total dos respondentes foram encontrados os seguintes resultados para o WHOQOL-bref:

Tabela 1- Distribuição dos graduandos da EPE-UNIFESP, participantes do estudo, segundo classificação de sua qualidade de vida

\begin{tabular}{|c|c|c|c|c|c|c|c|c|c|c|}
\hline \multirow[b]{2}{*}{ Série } & \multicolumn{2}{|c|}{ MR } & \multicolumn{2}{|c|}{$\mathrm{R}$} & \multicolumn{2}{|c|}{ NRNB } & \multicolumn{2}{|c|}{ B } & \multicolumn{2}{|c|}{ MB } \\
\hline & $\mathrm{n}$ & $\%$ & $\mathrm{n}$ & $\%$ & $\mathrm{n}$ & $\%$ & $\mathrm{n}$ & $\%$ & $\mathrm{n}$ & $\%$ \\
\hline $1^{\circ}$ ano & - & - & - & - & 9 & 4,9 & 24 & 13,0 & 4 & 2,2 \\
\hline $2^{\circ}$ ano & - & - & 3 & 1,6 & 16 & 8,6 & 48 & 26,0 & 5 & 2,7 \\
\hline $3^{\circ}$ ano & 2 & 1,1 & 7 & 3,8 & 11 & 5,9 & 16 & 8,6 & 3 & 1,6 \\
\hline $4^{\circ}$ ano & - & - & 1 & 0,5 & 7 & 3,8 & 25 & 13,5 & 4 & 2,2 \\
\hline Total & 2 & 1,1 & 11 & 5,9 & 43 & 23,2 & 113 & 61,1 & 16 & 8,7 \\
\hline
\end{tabular}


Tabela 2 - Distribuição dos graduandos da EPE-UNIFESP, participantes do estudo, segundo distribuição do nível de satisfação com a própria saúde, por série

\begin{tabular}{ccccccccccc}
\hline & \multicolumn{2}{c}{ MI } & \multicolumn{9}{c}{$\mathrm{I}$} & \multicolumn{2}{c}{ NSNI } & \multicolumn{3}{c}{ MS } \\
Série & $\mathrm{n}$ & $\%$ & $\mathrm{n}$ & $\%$ & $\mathrm{~N}$ & $\%$ & $\mathrm{n}$ & $\%$ & $\mathrm{n}$ & $\%$ \\
\hline $1^{\mathbf{0}}$ ano & - & - & 5 & 2,7 & 7 & 3,8 & 16 & 8,6 & 9 & 4,9 \\
$2^{\mathbf{o}}$ ano & 2 & 1,1 & 9 & 4,9 & 10 & 5,4 & 45 & 24,4 & 6 & 3,2 \\
$3^{\mathbf{o}}$ ano & 3 & 1,6 & 6 & 3,2 & 8 & 4,3 & 19 & 10,3 & 3 & 1,6 \\
$4^{\mathbf{0}}$ ano & - & - & 3 & 1,6 & 8 & 4,3 & 19 & 10,3 & 7 & 3,8 \\
\hline Total & 5 & 2,7 & 23 & 12,4 & 33 & 17,8 & 99 & 53,6 & 25 & 13,5
\end{tabular}

MI=Muito Insatisfeito/ I=Insatisfeito/ NSNI=Nem Satisfeito Nem Insatisfeito/ S=Satisfeito/ MS=Muito Satisfeito Fonte: Autoria própria (2009).

Tabela 3 - Escores máximo e mínimo, escore médio, desvio padrão e avaliação da QV da amostra, conforme domínios

\begin{tabular}{lccc}
\hline Domínios & Escore Médio (DP) & Mínimo & Máximo \\
\hline Físico & $64,2(15,1)$ & 21,4 & 96,4 \\
Psicológico & $64,5(15,3)$ & 8,3 & 95,8 \\
Relações Sociais & $69,3(18,4)$ & 8,3 & 100,0 \\
Meio Ambiente & $56,7(13,9)$ & 18,7 & 87,5 \\
\hline \multicolumn{4}{c}{$\mathrm{DP}=$ desvio padrão } \\
& \multicolumn{3}{c}{ Fonte: Autoria própria (2009). }
\end{tabular}

Fonte: Autoria própria (2009).

Do total dos respondentes foram encontrados os seguintes resultados para as variáveis sociodemográficas:

Tabela 4 - Distribuição dos participantes do estudo, segundo gênero

\begin{tabular}{ccccc}
\hline & \multicolumn{2}{c}{ Feminino } & \multicolumn{2}{c}{ Masculino } \\
Série & $\mathrm{N}$ & $\%$ & $\mathrm{n}$ & $\%$ \\
\hline $1^{\circ}$ ano & 34 & 18,4 & 3 & 1,6 \\
$2^{\mathrm{o}}$ ano & 67 & 36,2 & 5 & 2,7 \\
$3^{\mathrm{o}}$ ano & 37 & 20 & 2 & 1,1 \\
$4^{\circ}$ ano & 36 & 19,5 & 1 & 0,5 \\
\hline Total & 174 & 94,1 & 11 & 5,9 \\
\hline \multicolumn{3}{c}{ Fonte: Autoria própria (2009). }
\end{tabular}

Tabela 5 - Distribuição dos participantes do estudo, segundo renda per capita familiar mensal

$\begin{array}{ccccc}\text { Até um salário } & \text { Entre um e dois } & \text { Entre dois e três } & \text { Entre três e } & \text { Mais de quatro } \\ \text { mínimo } & \text { salários } & \text { salários } & \text { quatro salários } & \text { salários } \\ & \text { mínimos } & \text { mínimos } & \text { mínimos } & \text { mínimos }\end{array}$

\begin{tabular}{ccccccccccc} 
Série & $\mathrm{n}$ & $\%$ & $\mathrm{n}$ & $\%$ & $\mathrm{n}$ & $\%$ & $\mathrm{n}$ & $\%$ & $\mathrm{n}$ & $\%$ \\
\hline $1^{\mathbf{o}}$ ano & 2 & 1,1 & 2 & 1,1 & 11 & 5,9 & 13 & 7,0 & 9 & 4,9 \\
$2^{\text {o }}$ ano & 4 & 2,2 & 10 & 5,4 & 13 & 7,0 & 15 & 8,1 & 30 & 16,2 \\
$3^{\text {o }}$ ano & 4 & 2,2 & 4 & 2,2 & 5 & 2,7 & 14 & 7,6 & 12 & 6,5 \\
$4^{\text {o }}$ ano & 3 & 1,6 & 5 & 2,7 & 6 & 3,2 & 10 & 5,4 & 13 & 7,0 \\
\hline Total & 13 & 7,1 & 21 & 11,4 & 35 & 18,8 & 52 & 28,1 & 64 & 34,6 \\
\hline \multicolumn{8}{c}{ Fonte: Autoria própria (2009). }
\end{tabular}


Tabela 6 - Distribuição dos participantes do estudo, segundo quantas pessoas dependem da renda familiar

\begin{tabular}{|c|c|c|c|c|c|c|c|c|c|c|c|c|c|c|c|c|}
\hline \multirow[t]{2}{*}{ Série } & \multicolumn{2}{|c|}{$\begin{array}{c}\text { Uma } \\
\text { pessoa } \\
\text { vive da } \\
\text { renda }\end{array}$} & \multicolumn{2}{|c|}{$\begin{array}{c}\text { Duas } \\
\text { pessoas } \\
\text { vivem da } \\
\text { renda }\end{array}$} & \multicolumn{2}{|c|}{$\begin{array}{c}\text { Três } \\
\text { pessoas } \\
\text { vivem da } \\
\text { renda }\end{array}$} & \multicolumn{2}{|c|}{$\begin{array}{l}\text { Quatro } \\
\text { pessoas } \\
\text { vivem da } \\
\text { renda }\end{array}$} & \multicolumn{2}{|c|}{$\begin{array}{c}\text { Cinco } \\
\text { pessoas } \\
\text { vivem da } \\
\text { renda }\end{array}$} & \multicolumn{2}{|c|}{$\begin{array}{c}\text { Seis } \\
\text { pessoas } \\
\text { vivem da } \\
\text { renda }\end{array}$} & \multicolumn{2}{|c|}{$\begin{array}{c}\text { Sete } \\
\text { pessoas } \\
\text { vivem da } \\
\text { renda }\end{array}$} & \multicolumn{2}{|c|}{$\begin{array}{c}\text { Oito } \\
\text { pessoas } \\
\text { vivem da } \\
\text { renda }\end{array}$} \\
\hline & $\mathrm{n}$ & $\%$ & $\mathrm{n}$ & $\%$ & $\mathrm{n}$ & $\%$ & $\mathrm{n}$ & $\%$ & $\mathrm{n}$ & $\%$ & $\mathrm{n}$ & $\%$ & $\mathrm{n}$ & $\%$ & $\mathrm{n}$ & $\%$ \\
\hline $1^{\circ}$ ano & - & - & 2 & 1,1 & 7 & 3,8 & 18 & 9,8 & 7 & 3,8 & 2 & 1,1 & 1 & 0,5 & - & - \\
\hline $2^{\circ}$ ano & 4 & 2,2 & 6 & 3,2 & 7 & 3,8 & 36 & 19,5 & 14 & 7,6 & 3 & 1,6 & 1 & 0,5 & 1 & 0,5 \\
\hline $3^{\circ}$ ano & 7 & 3,8 & 2 & 1,1 & 6 & 3,2 & 12 & 6,5 & 8 & 4,3 & 2 & 1,1 & 2 & 1,1 & - & - \\
\hline $4^{\circ}$ ano & 4 & 2,2 & 1 & 0,5 & 7 & 3,8 & 17 & 9,2 & 6 & 3,2 & 1 & 0,5 & - & - & 1 & 0,5 \\
\hline Total & 15 & 8,2 & 11 & 5,9 & 27 & 14,6 & 83 & 45,0 & 35 & 18,9 & 8 & 4,3 & 4 & 2,1 & 2 & 1,0 \\
\hline
\end{tabular}

$\underline{\text { Tabela } 7 \text { - Distribuição dos participantes do estudo, segundo contribuintes da renda per capita familiar }}$

\begin{tabular}{|c|c|c|c|c|c|c|c|c|c|c|}
\hline \multirow[b]{2}{*}{ Série } & \multicolumn{2}{|c|}{$\begin{array}{c}\text { Um } \\
\text { contribuinte } \\
\text { com a renda }\end{array}$} & \multicolumn{2}{|c|}{$\begin{array}{c}\text { Dois } \\
\text { contribuintes } \\
\text { com renda }\end{array}$} & \multicolumn{2}{|c|}{$\begin{array}{c}\text { Três } \\
\text { contribuintes } \\
\text { com renda }\end{array}$} & \multicolumn{2}{|c|}{$\begin{array}{c}\text { Quatro } \\
\text { contribuintes } \\
\text { com renda }\end{array}$} & \multicolumn{2}{|c|}{$\begin{array}{c}\text { Cinco } \\
\text { contribuintes } \\
\text { com renda }\end{array}$} \\
\hline & $\mathrm{n}$ & $\%$ & $\mathrm{n}$ & $\%$ & $\mathrm{n}$ & $\%$ & $\mathrm{n}$ & $\%$ & $\mathrm{n}$ & $\%$ \\
\hline $1^{\circ}$ ano & 15 & 8,1 & 18 & 9,8 & 4 & 2,2 & - & - & - & - \\
\hline $2^{\circ}$ ano & 24 & 13,0 & 36 & 19,5 & 11 & 5,9 & 1 & 0,5 & - & - \\
\hline $3^{\circ}$ ano & 12 & 6,5 & 23 & 12,4 & 2 & 1,1 & 2 & 1,1 & - & - \\
\hline $4^{\circ}$ ano & 10 & 5,4 & 20 & 10,8 & 5 & 2,7 & 1 & 0,5 & 1 & 0,5 \\
\hline Total & 61 & 33,0 & 97 & 52,5 & 22 & 11,9 & 4 & 2,1 & 1 & 0,5 \\
\hline
\end{tabular}

Fonte: Autoria própria (2009).

Tabela 8 - Distribuição dos participantes do estudo, segundo apoio que recebeu dos pais e amigos para cursar enfermagem

\begin{tabular}{|c|c|c|c|c|c|c|c|c|c|c|c|c|}
\hline \multirow[t]{2}{*}{ Série } & \multicolumn{2}{|c|}{$\begin{array}{l}\text { Nenhum } \\
\text { apoio }\end{array}$} & \multicolumn{2}{|c|}{$\begin{array}{c}\text { Pouquíssimo } \\
\text { apoio }\end{array}$} & \multicolumn{2}{|c|}{ Pouco apoio } & \multicolumn{2}{|c|}{$\begin{array}{l}\text { Nem pouco } \\
\text { nem muito } \\
\text { apoio }\end{array}$} & \multicolumn{2}{|c|}{ Muito apoio } & \multicolumn{2}{|c|}{$\begin{array}{l}\text { Máximo } \\
\text { apoio }\end{array}$} \\
\hline & $\mathrm{n}$ & $\%$ & $\mathrm{n}$ & $\%$ & $\mathrm{n}$ & $\%$ & $\mathrm{n}$ & $\%$ & $\mathrm{n}$ & $\%$ & $\mathrm{n}$ & $\%$ \\
\hline $1^{\circ}$ ano & - & - & 3 & 1,6 & 4 & 2,2 & 11 & 5,9 & 4 & 2,2 & 15 & 8,1 \\
\hline $2^{\circ}$ ano & 1 & 0,5 & 3 & 1,6 & 5 & 2,7 & 11 & 5,9 & 25 & 13,5 & 27 & 14,8 \\
\hline $3^{\circ}$ ano & - & - & 4 & 2,2 & 1 & 0,5 & 6 & 3,2 & 11 & 5,9 & 17 & 9,2 \\
\hline $4^{\circ}$ ano & - & - & - & - & 2 & 1,1 & 5 & 2,7 & 7 & 3,8 & 23 & 12,4 \\
\hline Total & 1 & 0,5 & 10 & 5,4 & 12 & 6,5 & 33 & 17,7 & 47 & 25,4 & 82 & 44,3 \\
\hline
\end{tabular}

Tabela 9 - Distribuição dos participantes do estudo, segundo possuir ou não religião ou fé Possui religião ou fé Não possui religião

\begin{tabular}{ccccc}
\hline & \multicolumn{3}{c}{ Possui religião ou fé } & \multicolumn{3}{c}{ Não possui religião } \\
Série & $\mathrm{n}$ & $\%$ & $\mathrm{n}$ & $\%$ \\
\hline $1^{\circ}$ ano & 33 & 17,8 & 4 & 2,2 \\
$2^{\circ}$ ano & 64 & 34,7 & 8 & 4,3 \\
$3^{\circ}$ ano & 33 & 17,8 & 6 & 3,2 \\
$4^{\circ}$ ano & 33 & 17,8 & 4 & 2,2 \\
\hline Total & 163 & 88,1 & 22 & 11,9 \\
\hline \multicolumn{5}{c}{ Fonte: Autoria própria (2009). }
\end{tabular}

Tabela 10 - Escores e dados sociodemográficos significantes segundo domínios do WHOQOL-bref em graduandos da primeira a quarta série do curso de graduação em enfermagem da Escola Paulista de Enfermagem 


\begin{tabular}{ccccc}
\hline & Físico & Psicológico & Relações Sociais & Meio Ambiente \\
\hline Renda per capta familiar mensal & $\mathrm{p}<0,0325$ & $\mathrm{p}<0,0060$ & $\mathrm{p}<0,0300$ & $\mathrm{p}<0,0060$ \\
Quantas pessoas vivem da renda familiar & - & - & $\mathrm{p}<0,0106$ & $\mathrm{p}<0,0004$ \\
Contribuintes para a renda per capita familiar & $\mathrm{p}<0,0021$ & $\mathrm{p}<0,0495$ & - & $\mathrm{p}<0,0071$ \\
Apoio recebido de pais e amigos para cursar enfermagem & $\mathrm{p}<0,0001$ & $\mathrm{p}<0,0001$ & $\mathrm{p}<0,0001$ & $\mathrm{p}<0,0001$ \\
Religiosidade & - & - & - & $\mathrm{p}<0,0299$ \\
\hline
\end{tabular}

Significativo para $\mathrm{p}<0,05$, teste $t$ de Student.

Fonte: Autoria própria (2009).

\section{Discussão}

O maior percentual de participação foi encontrado na segunda série, seguido da terceira série. Este achado é semelhante ao encontrado em estudo sobre QV na graduação de enfermagem por Kawakame e Miyadahira (2005).

Os achados apontam que os graduandos de enfermagem avaliaram sua QV como boa (61\%), resultados semelhantes foram verificados em outros estudos (SAUPE et al., 2004; KAWAKAME. MIYADAHIRA, 2005; ARRONQUI et al., 2011).

Entretanto, tais resultados esbarram nos $30,2 \%$ de estudantes que classificaram sua QV de maneira negativa no presente estudo. Acredita-se que o ingresso na universidade atue sobre a QV, uma vez que, para a maioria dos jovens, isso acontece em uma fase de transição da adolescência para a vida adulta. Normalmente um período cheio de dúvidas que exige uma mudança no estilo de vida e necessita de um período para adaptação, a partir do qual estarão em uma nova etapa em que receberão novas responsabilidades (SCHERER; SCHERER; CARVALHO, 2006).

Quanto à percepção da própria saúde, observou-se que os alunos estavam satisfeitos (53,5\%), sendo os achados semelhantes ao estudo realizado em 2008 por Arronqui et al. (2011).

Ainda quanto ao WHOQOL-bref, o maior escore médio encontrado foi atribuído ao domínio relações sociais $(69,3 \%)$ e menor para meio ambiente $(56,7 \%)$ convergindo aos produtos apontados em pesquisa de QV de graduandos de enfermagem, no sul do Brasil, que obteve o maior escore médio no domínio físico e o menor no domínio meio ambiente (EURICH; KLUTHCOVSKY, 2008). Assim, concordam Eurich e Kluthcovsky (2008) e Beuter, Alvim e Mostardeiro (2005) que tais resultados negativos no domínio meio ambiente trazem preocupação uma vez que podem ser atribuídos ao ritmo capitalista, que impõe uma rotina frenética aos indivíduos, com supervalorização material e futurista.

De maneira semelhante, pôde-se observar que, os resultados concernentes às variáveis sociodemográficas são significativos na promoção da QV, uma vez que foram capazes de influenciá-la de modo diretamente proporcional. Mostrando-se semelhantes a estudos que descrevem a QV e o perfil sociodemográfico de alunos que cursam a graduação em enfermagem em outras instituições de diferentes municípios e estados (KAWAKAME; MIYADAHIRA, 2005; WETTERICH; MELO, 2007; MARQUES, 2006).

Por outro lado, a menor divisão da renda familiar poderia apresentar melhora na QV, contudo, no que se refere ao domínio das relações sociais, os resultados eram proporcionalmente melhores de acordo com o aumento do número de pessoas que viviam da renda familiar $(\mathrm{p}<0,0106)$, reforçando o entendimento de QV como subjetiva e pessoal, na qual se valoriza a opinião do indivíduo.

Todavia, o número de pessoas que possuiam aumento da renda familiar $(p<0,0060)$ e crença ou religiosidade $(\mathrm{p}<0,0299)$ proporcionaram uma elevação da QV para o dominio meio ambiente. Em estudo semelhante, demonstrou-se que, o aumento da renda e a crença favorecem as atividades de lazer na vida de acadêmicos de enfermagem, sendo promotoras da QV (BEUTER; ALVIM; MOSTARDEIRO; 2005).

Além disso, nota-se, na tabela 10 , que a QV é melhor quanto maior a renda per capita familiar em todos os domínios do WHOQOL-bref. Do mesmo modo, o nível de apoio recebido dos pais e amigos para cursar enfermagem mostrou-se significante nos quatro domínios estudados, melhorando a QV do aluno. Acredita-se que esses fatores possam influenciar o julgamento dos 
graduandos em relação à satisfação com as facetas avaliadas pelo questionário, com melhor rede de apoio e suporte em diversos aspectos de suas vidas, o que contribuiu para obtenção de resultados elevados nos diferentes domínios que compõem WHOQOL-bref.

Em contrapartida, em estudo de revisão, mostrou-se que situações de estresse, insegurança, volume de carga horária, inserção a pratica e relação professor-aluno foram os principais fatores capazes de influenciar negativamente a QV do graduando durante sua experiência acadêmica (ROSA et al., 2012).

\section{Considerações finais}

O presente estudo demonstrou que os alunos julgaram sua QV como boa e que aspectos sociodemográficos podem atuar significativamente sobre sua QV de maneira diretamente proporcional. Entretanto, entoa-se que quase um terço dos estudantes avaliou de maneira negativa sua QV, alarmando a carência de maior atenção quanto aos diversos aspectos de sua vida.

Variáveis sociodemográficas vinculadas aos aspectos financeiro, suporte e religiosidade demonstraram-se estatisticamente significantes na promoção da QV uma vez que foram capazes de influencia-la de modo diretamente proporcional, em todos os domínios avaliados pelo WHOQOLbref.

\section{Referências}

ALVES, E. F. Qualidade de vida de estudantes de enfermagem de uma faculdade privada. Revista Brasileira de Qualidade de Vida, Ponta Grossa, v. 2, n. 1, p. 23-30, jan./jun. 2010.

ARRONQUI, G. V. et al. Percepção de graduandos de enfermagem sobre sua qualidade de vida. Acta Paulista de Enfermagem, São Paulo, v. 24, n. 6, p. 762-765, 2011.

BEUTER, M.; ALVIM, N. A. T.; MOSTARDEIRO, S. C. T. S. O lazer na vida de acadêmicos de enfermagem no contexto do cuidado de si para o cuidado do outro. Texto contexto - enfermagem., Florianópolis, v. 14, n. 2, p. 222-228, jun. 2005.

EURICH, R. B.; KLUTHCOVSKY, A. C. G. C. Avaliação da qualidade de vida de acadêmicos de graduação em Enfermagem do primeiro e quarto anos: influência das variáveis sociodemográficas. Revista de Psiquiatria do Rio Grande do Sul, Porto Alegre, v. 30, n. 3, p. 211-220, dez. 2008.

FLECK, M. P. A. et al. Desenvolvimento da versão em português do instrumento de avaliação de qualidade de vida da OMS (WHOQOL-100). Revista Brasileira de Psiquiatria, São Paulo, v. 21, n. 1, p.19-28, mar. 1999.

KAWAKAME, P. M. G.; MIYADAHIRA, A. M. K. Qualidade de vida de estudantes de graduação em enfermagem. Revista da Escola de Enfermagem da USP, São Paulo, v. 39, n. 2, p. 164-172, jun. 2005.

MARQUES, M. K. E. et. al. O perfil do acadêmico de enfermagem da univap - são josé dos campos / sp que trabalha na área da saúde. In: X ENCONTRO LATINO AMERICANO DE INICIAÇÃO CIENTÍFICA, VI ENCONTRO LATINO AMERICANO DE PÓS-GRADUAÇÃO, v. 24, 2006, São José dos Campos. Anais... São José dos Campos: Universidade do Vale do Paraíba. 2006. p. 839-42. 
OLIVEIRA, R. A.; CIAMPONE, M. H. T. A universidade como espaço promotor de qualidade de vida: vivências e expressões dos alunos de enfermagem. Texto Contexto - enfermagem, Florianópolis, v. 15, n. 2, p. 254-261, jun. 2006.

OLIVEIRA, R. A.; CIAMPONE, M. H. T. Qualidade de vida de estudantes de enfermagem: a construção de um processo e intervenções. Revista da Escola de Enfermagem, São Paulo, v. 42, n. 1, p.57-65, mar. 2008 .

OMS (Organização Mundial de Saúde) 1946. Constituição. Disponível em $<$ http://www.onuportugal.pt/"oms.doc $>$. Acesso em 11 de junho de 2009.

ROSA C. S. et. al. Qualidade de vida dos graduandos de enfermagem: análise da produção científica. Revista Brasileira de Qualidade de Vida, Ponta Grossa, v. 04, n. 02, p. 38-49, jul./dez. 2012.

SAUPE, R. Qualidade de vida de estudantes de enfermagem conforme Escala de Flanagan. Ciência, Cuidado e Saúde, Maringá, v. 1, n. 2, p. 287-291, jul./dez. 2002.

SAUPE, R. et al. Qualidade de vida dos acadêmicos de enfermagem. Revista Latino-Americana de Enfermagem, Ribeirão Preto, v. 12, n. 4, p. 636-642, ago. 2004.

SCHERER, Z. A. P.; SCHERER, E. A.; CARVALHO, A. M. P. Reflexões sobre o ensino da enfermagem e os primeiros contatos do aluno com a profissão. Revista Latino-Americana de Enfermagem, Ribeirão Preto, v. 14, n. 2, p. 285-291, abr. 2006.

THE WHOQOL GROUP. The World Health Organization Quality of Life Assesment (WHOQOL): position paper the world health organization. Social Science and Medicine, v. 41, n. 10, p. 14031409, nov. 1995.

WETTERICH, N. C.; MELO, M. R. A. C. Sociodemographic profile of undergraduate nursing students. Revista Latino-Americana de Enfermagem, Ribeirão Preto, v. 15, n. 3, p. 404-410, jun. 2007.

WORLD HEALTH ORGANIZATION (WHO) et al. WHOQOL and spirituality, religiousness and personal beliefs (SRPB): report on WHO Consultation. Geneva; 1998.

WHO/MSA/MHP/98.2, 2-23), 1998.

Recebido em: 06 jun. 2013. Aprovado em: 30 set. 2013. 